\title{
EDUCACIÓN PARA LA LIBERTAD
}

\author{
De Javier $M^{2}$ Prades López
}

\section{Introducción}

T. S. Eliot se preguntaba con su persuasiva fuerza poética: $x_{2}$ Dónde está la vida que hemos perdido viviendo? / ¿Dónde está la sabiduria que hemos perdido en conocimiento? / ¿Dónde está el conocimiento que hemos perdido en información? $n^{2}$.

Nosotros podemos preguntarnos, parafraseándole, por qué nuestro deseo de educar pierde muchas veces por el camino esa sabiduria que hace vivir al otro y nos limitamos a transmitirle información que muchas veces no es capaz de evitar el fracaso del proceso formativo o, al menos, se limita a su realización en modo muy imperfecto. En una encuesta a 700 profesores universitarios españoles, realizada por la Asociación «Universitas» durante el año 2001, se reflejaba que un 94,3 de los encuestados consideraba que el fin de la Universidad debe ser aformar profesionales competentes", mientras sólo un 73,3 consideraba que fuese reducar al estudiante». Pues bien, además, sólo el 52,9 de los encuestados pensaba que en

\footnotetext{
'Charfa magistral realizada en el marcu del I Cungreso Internaciunal "Educaciôn parn la l.jberfad", UCSS, 24 y 25 de mayo del 2002.

Remite mas ampliamente a los siguentes textos: J. PRADES, walla iniziazione alla maturiti cristiana. Un itinerario di formazionon, Riscoprie Ja Confermurzione. Roma, Pontificinm Consilum pro Láás, 2000, pp.111-148, ID; $\alpha$ La ruptura de la visión unitaria del saber como tuptura del sujeto educativon, Asuciación prara la Investigación y la Ducencia Universias, Proyectus subre uAnálisis de las necesidades de formación del profesorado universitario y propucstas de mejuca a partir de: experiencias interuniversitarias y multidisciplinaresm. Madrid, 2001. Pro mumuscriłato, pp. 123 - 134

"T.S. ELIOT, "Coros de La Piedra" (D); Poesias remaidas Madnd, 1986. p. 169.
} 


\section{Javier Prades López}

la actual unversidad española se respondiera adecuadamente a esa finalidad de educar.

¿A quê se debe que casi la mitad de estos profesores universitarios piensen que no se logra educar al estudiante? ¿Por qué la generosidad y la buena intención de tantos esfuerzos, que no cabe desconocer, acaban en resultados insatisfactorios, tanto en la escuela secundaria como en la universitaria?, ¿en qué dirección nos conviene movernos?

A nadie se le escapa que el problema es todo, menos insignificante, puesto que está en juego la capacidad de nuestra generaciỏn de transmitir a los que nos siguen el bagaje de humanidad y civilización que hemos recibido. ¿H Habria que concluir precipitadamente que esa riqueza ha dejado de interesar a nuestros jóvenes o que la propia sociedad ha renunciado a ella, conformándose con format técnicos especialistas? Si la debilidad de la transmisión es indicio de una debilidad en la experiencia vivida debemos preguntarnos primero por las razones que dan lugat a una humanidad que en última instancia es poco interesante para el propio sujeto que la vive y la quiere transmitir.

Dado que cun pequerio error en el principio es un gran error al tinals, como decía Tomás de Aquino", nos meteriamos en un atolladero si atribuyéramos la dificultad sólo a una carencia en las técnicas pedagógicas, en los materiales o en los programas, o en las necesidades de financiación y nos limitásemos, en consecuencia, a buscat novedades en esos niveles de la formación. No son esos instrumentos y recursos -en si mismos imprescindibles para que exista la infraestructura universitaria, como es lógico- los que pueden devolver a la propuesta educaúva su interés para el hombre contemporánec.

Lo más importante en la educación no es un afjarre de educación, y todavia

'Cfr. Proyecto sobre uAmilisisian, o, c, pp.31:150.

"TOMAS DE AQUINO, De ente ef essentio, Proomminem

'1. MARITAIN, Per una filosofia dell"edurazione. Brescia, 2001, p. 86. Citado y comentado por A. SCOLA; una experiencia en acto: la Pontificia Unversidad Lateranensex: Asociación para la

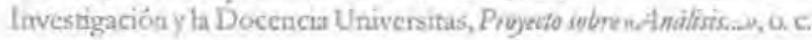




\section{EDUCACTONN PARA LA LIBERTAD}

menos de enseñanzay ${ }^{3}$. Con esta incisiva frase, Maritain va al centro de la cuestión educativa y reconoce la sorprendente paradoja de la que todo profesor, maestro o padre de familia es bien consciente. $Y$ el filósofo francés dice por qué: «la experiencia, que constituye el fruto incomunicable del sufrimiento y de la memoria, $y$ a través de la cual se lleva a cabo la formación del hombre, no puede ser enseñada en ninguna escuela ni en curso algunon. Algo semejante se refleja en la provocativa afirmación de alguien tan lejano del filósofo francés como es $\mathrm{O}$. Wilde: da instrucción es algo admirable, pero las cosas más importantes de la vida no se pueden enseriar, sólo se pueden encontrap). ¿Estamos con esto abogando por una enmienda a la totalidad, por una especie de abandono del sistema educativo y sus estructuras? De ninguna manera, perseguimos exactamente el propósito opuesto: queremos ver cuál es el mejor modo posible de hacerlo útil. Y para ello debemos situarnos en un punto de partida abierto a la totalidad de los factores, porque si en el arranque aceptamos una perspectiva reducida de lo que es educar, las posibilidades de respuesta convincente se evaporan. Debemos preguntarnos por la dinámica completa de la relación educativa entre docente y estudiante, entre maestro y discipulo, y en particular en el ámbito universitario.

\section{La educación como introducción a la totalidad de lo real}

2.1 Aunque la pedagogía se sigue ocupando seriamente del fenómeno educativo', no es facil encontrar una definición que sea a la vez tan comprensiva y tan sintética como la que ofrece Jungmann, cuando define, de modo elemental, el fenómeno educativo como "una introducción del hombre en la realidad totals".

\footnotetext{
Li, ci, p, 87 ,
}

Encre utrus crtudios remitimus a: G. JOVER, Reladön edtratiny y relationes bumamas. Barcelona, 1991 J. GARCIA CARRASCO, nEl concepto de educabilidad y el proceso educacional. Teoria de la Educaciónin: Revista lnteramiversianz (Salamanca), V, 1993, 11-32. P. BONAGURA, L'ate di invitare. II

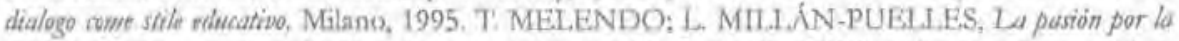
verdad. Hacia ana edvicación biberadora, Pamplona, 1997. I. M. BARRIO, Elementos de antropología pedigogice Madrid, 1998.

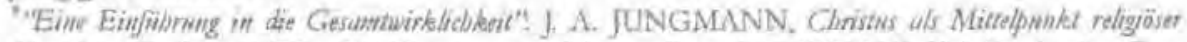

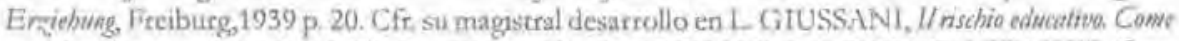
creaziane di personalita e di storia. Torno, 1995, p, 19ss. LGIUSSANL; S. ALBFRTO;J. PRADES, Crzar bmellas en la bistona dol mundo, Maurid, 2000". 


\section{Javier Prades López}

2.2 En esta definicion, el adjecivo «totals tiene un doble valor: se trata, por an lado, de desartollar las estructuras de la persona para que alcance su realización integral y. por otro, de que la persona esté en relación con rodas las dimensiones de la realidad. Realizaciôn total de si mismo en relación con la realidad entera: he aqui el fruto de un proceso educativo correcto, que podemos denominar también sunidad de la persona».

2.3 Como en Ia relación educativa se pone en juego el hombre a lo largo de todas las fases de su desarrollo respecto a la realidad entera, la educación acompaña al hombre durante toda su vida: el hombre estará siempre inmerso en un proceso educativo.

2.4 El motivo que justifica este carácter permanente de la educación proviene de la indole propia de la razón humana, entendida como sconciencia de la realidad según la rotalidad de sus factoresw. En efecto, el hombre es un buscador infatigable, que vive en una tensión de apertura a todos los aspectos de la realidad, intentando comprender sus relaciones entre si y en su conjunto. En esta búsqueda, es el impacto con las cosas el que despierta las exigencias de la razón y aviva cada vez más el deseo de conocer la propia vida y la realidad circundante. Dadas estas características de la razón, el hombre no se pacifica mientras no llega a descubrit el significado de las cosas, ya que no hay posesión humana de la realidad si no va acompañada de su significado completo, Mientras realiza cada una de sus acciones, el hombre tiende inevitablemente, aunque muchas veces no lo piense, a un horizonte más amplio y más profundo que le dé el significado completo de aquel aspecto particular en el que está comprometido, como apuntaba magistralmente Dante: "Cada cual concibe confusamente un bien en el cual el alma se complace y lo deseas por lo cual, todos luchan por alcanzarlos ${ }^{\text {tii }}$. El

\footnotetext{
"Sobre la admiración ante tí realidad cano mucio del conocimiento se pueden ver ARISIÓTEL.FS,

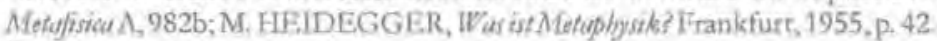

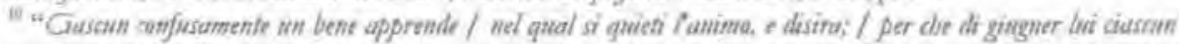
pontende". DANTE Al.tGHIERI, Prrgatorio, Canto XVII, vv.127-129. La definición del hornbre como buscadur de la verdad recurre la Encíclica Fides of Rutio (cfr. en particular N." 24. ss.). Una profundikima reflexićs sobre la nanuraleza propia de la verdad, su oniginaria relación cun ta Libertady el misterio, asi como su fundamento en d amor, se encuentra en H U. VON BAITHASAR, Teologer, vasl. 1 (= Ventud del mintido), Madrid, 1997.
} 


\section{EDUCACTÓN PARA LA LIBERTAD}

progreso imparable de las ciencias y de la vida social muestra bien a las claras cómo la actividad humana se ve siempre empujada, en viurud del carácter racional del sujeto, hacia un horizonte de apertura total ${ }^{11}$.

2.5 El hombre no entra en esta relación con la realidad a partir de cero, por así decir, sino que su punto de arranque es la tradición humana en la que ha nacido y que le ofrece una primera hipótesis de interpretación de cada descubrimiento que hace al vivir. No está obligado a rehacer trabajosamente por completo cada aspecto de la vida social, cientifica o humanista, sino que se beneficia de muchos conocimientos que le facilitan su propio e inalienable esfuerzo personal. La tradición aparece pues, como un componente ineludible del progreso humano en el conocimiento de la realidad ${ }^{12}=$

2.6 Existencialmente se concreta por medio de la relación maestro-discípulo (educador-educando). En efecto, cuando se está ante una personalidad rica en algún aspecto de la vida (un gran deportista o un gran médico o un gran profesor.-) pero, sobre todo, cuando se encuentra a una personalidad rica en la sabiduría misma de la vida, se produce esa impresión de novedad que despierta la curiosidad, la atención, el respeto y nos dispone a conocer. La capacidad de apertura a todas las dimensiones de lo real y la exigencia de alcanzar la verdad se ven asi facilitadas, porque estos dinamismos se ponen en movimiento ante la proyocación de alguien que aparece a los propios ojos como una autoridad en el sentido etimológico del término (autoritas de angeo, incrementar, hacer crecer).

Basten estos rasgos para una definición provisional del fenómeno educativo, que nos permita orientarnos en nuestro recorrido. La educación entendida como introducción a la totalidad de lo real es un proceso que dura toda la vida, en virtud de la racionalidad del ser humano que se abre a la infinita realidad, $y$ su modalidad

\footnotetext{
"J. H, NEWMAN describio la actividad de la razón en estos térninos: wla capacidad de ver las cosas sumultáneamente como una tutalidad, de remitirlas a su lugar adecuado en el sistema universal del saber, de comprender su valor respectivo $y$ determinar su dependencts xeciprocan. The idea of a Umiversits. New Haven-London, 1996, p. 99. Sobre la capacidad humana de llegar a una visión unitana y orgánica del saber, cfr. también FR N, ${ }^{\circ} 85$.

${ }^{12}$ Cfr. FR N. 31.
} 


\section{Javier Prades López}

existencial requiere el encuentro con un umaestro", es decir, con alguien en quien se reconoce ya realizado aquel valor que se desea adquirir. Es más, sólo cuando el maestro al que se sigue es "el único Maestrow" entonces la introducción en la realidad es definitiva y la adhesión a Él sacia por completo la exigencia de racionalidad $y$ libertad tipica del hombre.

La tradición bimilenaria de la Iglesia ha acumulado una experiencia educativa en todos los campos del saber humano de tal manera que, con su vida entera, la Iglesia ha educado, es decir, ha comunicado el significado efectivo de la existencia.

\section{El proceso de secularización y sus consecuencias sobre la educación}

3.1Y, sin embargo, como veíamos al examinar los datos de la encuesta, parece que hoy la efectiva educación de los estudiantes cede cada vez más su lugar a una mea concepción técnico-formativa de la vida universitaria para una capacitación profesional inmediata. No queremos desconocer todas las ventajas de una estrecha relación entre vida universitaria y vida profesional-laboral. Pero si hacer notar las profundas consecuencias que se siguen de una concepción educativa que senuncia al horizonte de totalidad que hemos señalado ${ }^{14}$. Una transformación así de la idea de educar tiene que verse en estrecha relación con un cambio en la concepción del sujeto educador, del hombre. ¿Cómo se ha podido llegar a una época que teoriza la ruptura de la unidad y totalidad del saber y del sujeto y que lleva a una situación como la presente?

3.2 La historia del pensamiento y de la cultura occidental en los últimos siglos pone de manifiesto una transformación que ha a fectado profundamente tanto a la vida de los hombres como de las sociedades y con ello también a la vida y la misión de la Iglesia. La interpretación de este fenómeno culrural y teológico, que se suele denominar «secularnzación», ha hecho correr rios de tinta y desborda los limites de

\footnotetext{
"Cfe Mt 23,8.

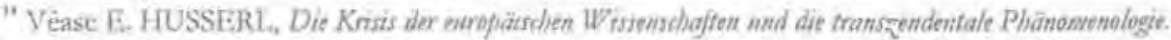

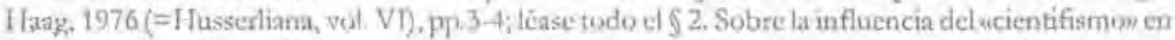
L cencepción misma de los estudios unversitadios y la distiación entre cultura y ciencia, efr. J. ORTEG Y Y GASSET, eiMisión de La Unuversidadis en Obnas Camplefies, Madrid, 1983, vol. 4,pp. 313 353.
} 


\section{EDUCACIÕN PARA LA LIBERTAD}

nuestra intervención. No es ahora posible abordar tan enorme problemática en profundidad. Baste indicar que en este complejo proceso van a la par la mutación de la concepción del hombre y la alteración de su relación con Dios, tal y como la tradición cristiana la había vivido y propuesto.

En efecto, por una parte et hombre moderno se concibe a si mismo de tal manera que cada vez es menos capaz de conocer la realidad que está ante él, porque la razón y la libertad oscurecen progresivamente su apertura característica a lo real.

Por otra parte, también se reinterpreta el hecho cristiano y se le atribuye una naturaleza diferente a la que la tradición eclesial le ha reconocido siempre. El cristianismo deja de ser visto como la permanencia en la historia del acontecimiento vivo de Cristo, del que se puede tener experiencia en el presente (visión de la tradición), y pasa a ser mera expresión de religiosidad o de ética, apoyada en un recuerdo del pasado, aunque, por desgracia, en la modernidad no han faltado tampoco los que califican a la fe cristiana fout court como una invención al servicio de intereses espurios ${ }^{15}$.

$\mathrm{Y}_{\mathrm{a}}$ en los primerisimos diálogos de los cristianos con el mundo cultural circundante argüian que la comprensión del hombre y la del acontecimiento cristiano se reclaman mutuamente. 'Teófilo Antioqueno, en un texto bien conocido, rebatía: «Si tú me dices: "muéstratne tu Dios", yo te diré: "muéstrame tu hombre y yo te mostraré mi Dios" hombre y reinterpretación del hecho cristiano, caminan a la par en los últimos siglos de la historia del pensamiento occidental, en ese proceso complejo que se suele denominar useculatización» y que De Lubac califica como un "trágico equívoco ${ }^{17}$. El resultado al que se llegará, to sólo en la mentalidad dominante, sino también, como deciamos, en el reflejo que deja en nuestras conciencias, puede identificarse con el estribillo que solia repetir Cornelio Fabro: wio se c'è non c'entras.

\footnotetext{
"Sobre este aspecto del problema yoiveremor más abaju, en el apactado 5

${ }^{16}$ TEOFILO NNTIOQUENO, Ad Awolycm 1, 2.

"Cfr. H. DE LUBAC. El drama del lismomisone ateo. Madrid, 1967, pp. 17-25. La bibliografia sobre la relación cornpleja entre modernulad y secularizsción es inabarcable Entre les dásicos: $\mathrm{T}$
} 


\section{Javier Prades López}

\subsection{La mutación en la comprensión de la razón y la libetrad del hombre}

3.3.1 Porlo que respecta a la reinterpretación del hombre, la actitud que nace de la modernidad se apoya en un presupuesto tnamovible: el hombre está dotado de una capacidad de saber y poder que no admite ninguna instancia superioc. Se rechaza cualquier remisión más allá de sí mismo y se afirma la plena correspondencia unatutals entre el hombre y su ideal cognoscitivo y ético ". Para comprender el origen de esta actitud es necesario apuntar, con suma brevedad, a los cambios que la razón y la libertad han sufrido en este periodo. No se puede comprender la transformación del hombre (cristiano) al margen de los presupuestos epistemológicos $y$ ontológicos que definen la época moderna.

3.3.2 El nacimiento de la modernidad conlleva una exaltación insólita del sujeto humano como principio absoluto. El sujeto se va a erigir en un punto de referencia último de cualquier afirmación que pretenda tener validez. Hegel definirá comó problema del cauto-cerciommientom esta pretensión de que el hornbre se constituya en un sujeto que busca en si mismo el fundamento inalterable de la propia representación. El esfuerzo especulativo desde Descartes a Kant y Hegel intentari ofrecer la legitimación teórica de la pretensión de erigir la subjetividad como el lugar y el fundamento de todo significado. La exaltación teórica del sujeto irá acompanada por la idea que puede también disponer sin limites de si mismo y de las cosas: conocer más significa tener más poder (Bacon). A esta raiz podemos vincular otros factores que caracterizan la modernidad, como soa el desarnollo de la macionalidad científico-técnica o la voluntad de autonomia.

El típo de saber que nace con la modernidad se basa en una concepción absoluta de la razỡn. Cuando decimos que la razón es absoluta, en sentido etimológico (absoluta), queremos subrayar dos aspectos que van unidos: por un lado la razón pone el fundamento del saber solo en si misma, separada del acto con el que la

ILAZARD, La crises de la comienca muruph, Madrid, 1988 (oc. 1934). ID, E/ pensamiento wropeo en ol sesto X7/II. Madrid, 1985 for 1946), R, SUUARDINL, El ocuso de la Edad Moulerne, Madrid, 1981 (ur 1950). Otrus estudios: G. COY'TLER, Questions de la Modertite, Paris, 1985. M. UREŇ́, J PRIDES (Eds). . Hombre y Dios en la seciedud de fin de sugho. Madrad, 1994.

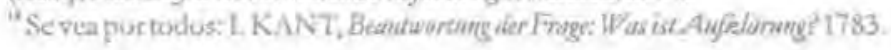




\section{EDUCACIŌN PARA LA LIBERTAD}

conciencia se refiere a la realidad, $y$, por otro lado, se considera absoluta en cuanto se considera horizonte totalizante y completo de todo saber, hasta llegar a excluir del ámbito racional todo lo que no entre en la medida que la propia razón constiture y determina.

3.3.3 Ahora bien, la consecuencia de esta forma de entender la razón y la subjetividad es que se va a ir perdiendo el sentido de la presencia de lo real. Tras el giro antropológico cartesiano no se niega todavia groseramente la existencia de la realidad exterior a la conciencia - aunque se llegará al final a posiciones claramente nihilistas - sino que, más bien, das cosas son abordadas por el hombre no con la intención de dejarse instruir por ellas sobre lo que ellas son en si mismas (como sujeto o sustancia - wbjectum, suppositum-), sino a título de objeto susceptible de corresponder al designio que él piensa que lleva en si mismo en cuanto sujeto únicon". En este proceso, la realidad del mundo se debe reducir a la representación que el sujeto se hace de ella, y para lograr este fin es necesario que ese sujeto se cierre cada vez más a la misteriosa trascendencia del ser. La realidad se ve asi privada de su prerrogativa más genuina, que es ta de ser portadora de un significado que la desborda. Dicho de otro modo, la realidad deja de ser el signo del Misterio que la hace y la sostiene. Hegel reconoce que đa reducción de la religión al ámbito de la subjetividad conduce a la superficialización de la realidad, por una parte, $y$ al vaciamiento de la religión, por otra. El mundo pierde a Dios y Dios pierde al mundo y queda sin objeto, en el sentido propio de la expresión. Las consecuencias son el atefsmo y el nihilismon ${ }^{20}$. El proceso de la modemidad llegará a repudiar cualquier presencia que coloque al sujeto en una posición de dependencia: primero se excluye la presencia de Dios y al funal la presencia misma de las cosas. Mientras que, para Tomás de Aquino, entender no es mis que tener algo presente, es decir, la presencia inrencional del ser ${ }^{2}, y$ de este modo establece un vinculo inescindible entre razón y realidad, la razón modema ya no reconoce el

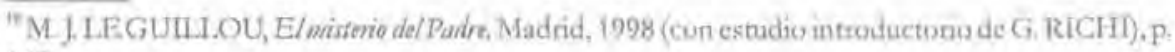
160.

37. C.F. W KASPER (E/ Dios de jesurrish Salananca, 1985, p. 32) que remite a GWW HEGEL, Glumben und Wissen (WWI, ed. Glockner, 433).

" shntelligere vihil aliud est quam aliquid priesentialiter haberes: TOMASS DE. AQU1NO, Scriptum super Senteutris, Id, 17 a. 4 add
} 


\section{Javier Prades López}

ser y su verdad sino que los produce ${ }^{22}$, La consecuencia en la que se desembocatá es la negación del valor de la realidad tanto en su presencia como en su significado (carácter de signo). La famosa escena de La náxsea de J. P. Sartre nos ayuda a comprobarlo: $\mathrm{xN}_{\mathrm{N} i}$ siquiera podia uno preguntarse de dónde salia aquello, todo aquello, ni cómo era posible que existiera un mundo mejor que nada. Aquello no tenia sentido... No había habido momento en que hubiera podido no existir. Eso era lo que me irritaba: clato que no habia ninguna razón para que existiera esa larva resbaladiza. Pero no era posible que no existiera... La sonrisa de los árboles, del macizo de laurel quería decir algo; aquel era el verdadero secreto de la existencia... Parecía como si las cosas fueran pensamientos... que permanecian asi, saltando con un sentido pequeño y ridiculo que las excedía. Este pequeño sentido me irritaba.. Me fuin ${ }^{23}$.

3.3.4 Pues bien, la máón moderna que se rebela contra la presencia no ya de Dios sino del significado de la realidad, no se convierte en la duefia que pretendia ser y acaba presa de si misma, en el juego indeterminado de las mil y una autointerpretaciones que ha traido la posmodernidad. Haciendo gala de humor británico, Chesterton decía que sel loco no es el que ha perdido la razón sino el que ha perdido todo menos la razóns $\rangle^{24}$, para hacernos entender que la mayor grandeza de la razón es precisamente su capacidad de reconocimiento de la realidad total, como emerge en la experiencia del hombre. Por eso, ha dicho certeramente J. Guitton que «(razonable) es someter la razón a la experiencia»?".

3.3.5 Algo parecido sucede con el otro dinamismo típico del ser humano, que es la libertad. En nuestros dias se la exalta como dueria y señora absoluta, usurpando así el protagonismo que le correspondió a la razón en la modernidad, pero también la libertad acaba exasperada y se agota en sí misma al intentar realizarse,

\footnotetext{
"Mientras que los escolásticos decían: verum est ens, los tacdernos (Vico) dirác: verum quia factum, que desembocara cn el veruen quia fuciendum. Cfr. J. RHTZINGER, Introducrión al mistatumsmo, Salamanca, 1982 , pp $38-48$.

"I. P.SARTRE, Lo nutwea. Buenuss Aires, 1977,pp. 152-153.

G. K.CHESTIERTON, Ortodossid, Brescia, 1980, p.27.

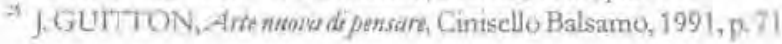




\section{EDUCACIŌN PARA LA LIBERTAD}

ya que la realidad que podria saciarla ha perdido toda su conststencia y el impetu infinito del deseo no logra encontrar un objeto adecuado que lo satisfaga. Si las cosas se reducen a su apariencia, para dominarlas exhaustivamente sin que nada escape al control de la medida humana, ya no entregan su misterio, que es el ser, signo de la única realidad que sí puede aquietar la exigencia in finita de la libertad: el Misterio.

Podemos decir, en resumen, que el hombre moderno concibe la razón y la libertad como una medida y por eso son sus propias fuerzas las que deciden lo que es posible a no lo es. La realidad se reduce, consecuentemente, a una pura representación dependiente del poder y el saber del sujeto. El hombre pierde su relación con la realidad y, por tanto, no sabe ya qué hacer con su razón y su libertad para explicar su destino, a pesar de los ingentes progresos de orden técrico y cientifico que ha producido la razón instrumental.

\subsection{La fragmentación del proceso educativo}

3.4.1 La parábola que ha descrito el pensamiento moderno y posmoderno tiene consecuencias evidentes para la tarea educativa. No es misión nuestra entrat en un estudio detallado del panorama de las ciencias pedagógicas; baste con indicar que uno de los resultados más notorios de su aplicación es la actual fragmentación de la educación, es decir, el efecto opuesto a la exigencia de unidad y totalidad que caracteriza una educación bien lograda.

3.4.2 Esa fragmentación aparece, en primer término, cuando se excluye la unidad del saber: se considera que es imposible una relación entre las distintas disciplinas científicas y humanistas no sólo de facto. por la creciente complejidad de datos disponibles en cada área del conocimiento, sino de jurt, porque desaparece una forma de entender la razón que esté en condiciones de abarcar todas las dimensiones de la realidad ${ }^{\text {th }}$. En el ámbito de las ciencias humanas, por ejemplo, se cede cada vez más a la yuxtaposición de una serie indefinuda de purtos de vista parciales -ofrecidos por los expertos; psicología, sociologia, antropologia,

\footnotetext{
${ }^{2}$ Para una descripciún de la ppesteme posmoderna etr: 1. MUNO\%, "Inventario provisional (modernos, postmodernosy antimodernos)wen Rewsto de.Ocadente 66. (1986), Pp. 5.22.
} 


\section{Javier Prades López}

pedagogia... - que renuncia a buscar el punto sintético e irreductible a cada una de esas perspectivas, el significado úldimo en relación con la totalidad ${ }^{27}$.

3.4.3 Pero, en realidad, esta fragmentación de las disciplinas académicas denuncia en su raíz una fragmentación del sujeto, prisionero de una razón absolutizada y, por tanto, cada vez más incapaz de usar su propia razón para conocerse a símismo y a la realidad que le circunda según todas sus dimensiones. El proceso del saber en la modernidad, al acentuar la «auto-fundacións en el propio sujeto, con la intención de evitar el peligro de la incerteza, mortifica una dimensión constitutiva de la razón como es su receptividad, es decit, su capacidad para acoger los objetos del conocimiento de tal manera que el sujeto se implica en ellos, los recibe en unidad y se incrementa su propio $\sec ^{28}$. El poeta Juan Ramón Jiménez lo evocaba bellamente: "Todos los dias, yo soy yo, pero ¡qué pocos dias yo soy yo! [...] $\mathrm{iVe}$ despacio, no corras, que el niño de tu yo, recién nacido eterno, no te puede seguitls ${ }^{21}$. La acumulación de conocimientos parciales no se traduce en una maduración, en un incremento de la personalidad de quien adquiere tales conocimientos. El saber se cultiva entonces como instrumento de dominio, al servicio del propio poder, y la educación se reduce a proceso de adiestramiento en el manejo de capacidades. El conocer no tiende ya al reconocimiento de la realidad como signo, como un rdaton que proviene de otro y es portadora de un significado irreductible a la propia precomprensión, ni mucho menos, tiende a la unidad amorosa con esa realidad y su significado. El filósofo espanol X. Zubiri exigc, por ello, la apertura simultánea a la realidad y al sentido de los hechos, como punto de partida de una educación verdadera. kAl enfoque racionalista de la educación intelectual, cuyo ideal consiste en un enseñar a pensar que se identifica con enseñar a conocer verdades discursivamente de acuerdo con normas lógicas, se opone Zubiri por entender que la función discursiva no puede ser lo primero, ni en lógica ni en pedagogia, ya que todo razonamiento válido parte y se apoya en

\footnotetext{
27 Filles et Ratio advierte contra un cierto abuso de las wciencias humanasn, cuya metodologia empírica sólo puede obtener resultados hipotéticos dentra de su àmbito, con la intención de sustituir a una Filosofia del ser, capaz de plantearse la cuestion de la verdad ( $\left(0^{\circ}, 61,69\right)$.

${ }^{3}$ Cfr A. SCOIA Ospitare il reale Roma, 1999, p. 65. Más ampliamente H.U. VON BAIJHASAR. Teologica, vol. 1.0.c.

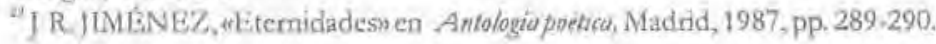




\section{EDUCACIÓN PARA LÁ LIBERTAD}

una previa percepción de los objetos. Por eso en la educación debe enseñarse, antes que a demostrar verdades, a mirar con los sentidos y a comtemplar con la inteligencia los objetos inmediatamente dados, para poder intuir en ellos sus propiedades sensibles e inteligibles. "El verdadero educador de la inteligencia", sostiene Zubiri, "es el que enseña a sus discipulos a ver el "sentido" de los hechos, la "esencia" de todo acontecimientos".

Las cosas $-\mathrm{y}$ no pocas veces las personas - se estudian y se describen como objetos dentro de un proyecto cuya finalidad no parece ser otra que ver hasta dónde llegan las aptitudes técnicas o cientificas del hombre. En lugar de unidad con lo real, se busca someter lo real, en un doble sentido: como autoafirmación de un poder que no admite límites $y$, consecuentemente, como autodefensa del hombre frente a la sospecha de que lo real pueda constituir una amenaza a ese intento de poder ilimitado ${ }^{31}$. En ese camino que tiende a hacer del hombre tan sólo un experto en algún campo del sabet o la técnica, cabe preguntarse si queda margen para buscar aquel conocimiento que no termina en información sitio en sabiduria. El dramaturgo siciliano Pirandello se sorprendia de que wnadie tiene ni el tiempo ni la ocasión de pararse un momento para considerar si lo que ve hacer a los demás, lo que él mismo hace, es lo que verdaderamente le conviene por encima de cualquier otra cosa, lo que le puede dar aquella única certeza verdadera en la que encontratia su descansos? ${ }^{32}$. Una educación que surge de una razón instrumental pierde la capacidad de preguntarse por lo que verdaderamente conviene, y no busca la certeza verdadera.

3.4.4 El sujeto que se uabsolutiza» acaba padeciendo otra manifestación de esa fragmentación que es su separación respecto del otro. El hombre moderno aparece

\footnotetext{
91. A. MARTÍNEZ, sLa educación según Xavier Zubirnen El Pais, 4-XII-1998.

ir Comer la pedagogia americana ha mareado tan decisivamente las líneas de la renovación pedagúgica también en Europa, no estará de más recurdar que uno de sus inspiradures más celebrados, J. Dewey, està en estrecha relación con Foucault, James y Nietasche precisamente por wel sentida de que no hay nada en las profundidades de nosotros mismos que no bayamos puesto nosotross, según expresión de R. Rorty, Cfr. E. BUZZZI, wJohn Dewcy: la maturaleza sin destinom en Rrvistu Católica Internacianal Commmio 17, 1995. pp. 545-554.

${ }^{2}$ L. PIRANDELLO, wQuaderni di Serafino Gubbio vperattrer, Quaderno primu, 1 en Tuthi $I$ ronsangi. Nilano, 1994 Vol, II, p. 520
} 


\section{Javier Prades L.ópez}

aislado, como resultado de su des-vinculación de la realidad y de su desvinculación de los otros, cuya consistencia es puesta en duda. De este modo se desconoce, con graves consecuencias, que en el sujeto espiritual está implicada intrinsecamente la relación interbumana, en cuanto que el kyom se descubre a sí mismo sólo en la modalidad intersubjetiva con la que se le abre el mundo, a través de la interpelación del otro. La autoconciencia libre se descubre como kyom al sentirse llamada y tratada como un atús, y con ello admitida en la comunidad. Es la palabra «pertenencias la que define este rasgo del hombre, que tiene una consistencia propia irreductible, pero cuyo inicio no está en uno mismo como principio absoluto. Cada hombre llega a descubrir su constitutiva relacionalidad con el otro, que funda lo que podemos llamar el carácter sresponsorial» de la vida $^{33}$.

\section{Los efectos en la vida universitaria}

Las consecuencias de este proceso de fragmentación en el ámbito universitario son muy variadas. Me limito a indicar tres que considero especialmente relevantes.

4.1 En primer ligar, lo que podemos llamar intelactualismo, esto es, la comunicación de un saber cientifico que se lleva a cabo de modo abstracto, es decir, separado de la vida concreta del sujeto que enseña y del sujeto que aprende. Dicho en términos banales, es la actitud de quien a partir de varios libros escribe otro, entremezclando las ideas de los demás y ariadiendo alguna crítica. Ante este riesgo, que siempre sentimos cercano, agradecemos la posibilidad de usar la razón sometiéndola a la experiencia, como decía Guitton, de aprender a descubrir la transparencia misteriosa de lo real, tal y como se da en la experiencia.

4.2 La segunda es el individualismo académico, entendido como la deferrsa celosa de la producido autonomamente, que se erige en barrera frente al otro (dominio, instrumentalización...) y no en cauce de comunicación. Sin embargo, hay un modo de compartir el patrimonio intelectual e ideal en la tarea universitaria por el

\footnotetext{
"Cfr. más ampliamente f. PR/DES, wsius dulas Pracsentà. Nutas sobre el aeceso del homber al misterio de Diosin en Rrivista Espaniula de 7evlogia 02, (2002).pp 5-44
} 


\section{EDUCACIÓN PARA LA LIBERTAD}

que uno reconoce que recibe más de lo que da, y esa gratitud estimula tuevamente una condivisión de lo que se encuentra personalmente;

$4.3 \mathrm{Y}$, en fin, la pérdida del sentido de la vida universitaria como vocaciön personal. Si el sujeto cognoscente está «separado» de sus conocimientos, puesto que no los «recibes en una asimilación que le haga crecer, y está separado de los otros miembros de la comunidad académica, el trabajo de investigar y enseñar no puede coincidir con la maduración de la propia vida, con su cumplimiento, ya que el trabajo fragmentado en múltiples aspectos carece de un punto sintético unitario que lo ponga en relación con la totalidad de dimensiones de la persona.

\section{La secularización en cuanto reinterpretación del hecho cristiano afecta a su capacidad educativa}

5.1 Esta reinterpretación del hombre va acompañada, en la modernidad, de una reducción a la hora de comprender la naturaleza del cristianismo. Ambos procesos se han dado simultáneamente en el tiempo y actúan el uno sobre el otro. No podemos explorar aquí todos los recodos de un itinerario que ha sido largo y sinuoso. Nos limitamos a enumerar algunas etapas de esta reinterpretación del cristianismo en la modernidad, cuyos precedentes se remontan hasta el renacimiento y la baja edad media ${ }^{34}$. En una primera fase se puede hablar de una «reducción del cristianismo al ámbito privadom, en cuanto que la fe se ve limitada respecto a la razón (Descartes) y se reduce su pretensión de influencia en el ámbito social y político (Hobbes-Rousseau, Locke). El paso siguiente scrá una verdadera «reinterpretacións del hecho ctistiano, primero en términos éticos (ilustración de Lessing y Kant) y después reinterpretado como cosmovision inmanente (Hegel). La etapa ulterior llevará al (ateismo» explicito de aquellos que consideran el cristranismo una ilusión bajo la que se perpetuan y se enmascaran situaciones de opresión o de resignación (Feuerbach, Marx, Nietzsche). Si en los primeros momentos, el cristianismo reducido o reinterpretado todavia habia

\footnotetext{
" Cf. F. BOTTURI, de tappe della secularizzazionen en M.VV La Chesa del Candio, Milanox. 1985, pp.153-164. 1D, "Modernità e crisi dell'umversale: dalla secolarikxartone al nuchitikmon con Pm la filosofio, Milane, pp.82-91.M. BORGIIES1. Posmodernidad y crishomismo, Madrad. 1997
} 


\section{Javier Prades Lōpez}

podido ser exaltado como humanismo, al final se le juzga rigurosamente como antihumanismo.

5.2 En este proceso no se habia negado enseguida a Dios, simplemente habia ido desapareciendo como factor real de la experiencia humana. Tan sólo en un segundo momento, cuando ese Dios inútil llegue a ser percibido como un obstácula para la plena autonomía de realización del hombre, será eliminado para dejar espacio a la razón y la libertad absolutas que buscan su satisfacción.

5.3 Una consecuencia muy importante de este recorrido es que las realidades de la feno escapan a las reglas que impone una subjetividad humana estructurada en los rérminos vistos. Fl hombre creyente, que absorbe cada vez más los planteamientos de la mazón moderna, va colocando la fe en el espacio que le deja libre la razón. Ahora bien, puesto que la razón moderna es cabsolutay, es inevitable que la fe sea exterior a la razón, y con ello termine situada en los márgenes de la realidad que la razón conoce y domina: sólo le quedarán el sentimiento o la pura interioridad. Ya no es una modalidad (por gracia) de conocimiento $\mathrm{y}$ de adhesión a la realidad, coma se la había entendido tradicionalmente ${ }^{35}$. Este fenómeno ha sido denominado «extrinsecismo» o "dualismo» entre fe y razón, y en él la fe aparece como un añadido o una superposición a la razón con funciones meramente correctoras". Guardini describe de este modo el cristianismo dividido y frágil que caracteriza la ćpoca moderna: «Entonces [después de la concepción unitaria medieval] comienza la separación. La filosofia se separa de la teología, la ciencia empirica de la filosofia, la indicaciones prácticas del conocimiento del ser. Esta preocupación que podia tener su legitimidad... es también peligrosa porque profundiza y cristaliza la raptura espiritual del hombre moderno. Lo que nos importa aqui es que la unidad de la conciencia también se fragmenta en los cristianos. El creyente ya no está con su fe dentro de la realidad del mundo, asi como, por otra parte, tampoco se puede encontrar el mundo dentro de su fes ${ }^{37}$. Algo parecido habia diagnosticado

\footnotetext{
"Para TOMÁS DE XQUINO, por ejemplo, es pacifico que la fe es uns forma de conocimiento, que

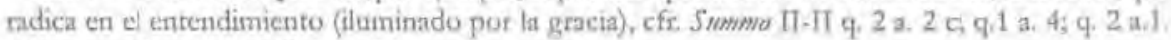
Véase también FRN. ${ }^{\circ} 43$.

"Cfr. G. COLOMBO, Langgome seolqgico, Milano, 1995, Véase FR N."45.
} 


\section{EDUCACIÓN PARA LA LIBERTAO}

agudamente J. H. Newman respecto al cristianismo del siglo XIX: «La religión, en cambio, siendo algo personal, debiera ser real, pero exceptuando un pequeño numero de personas, ordinariamente no es real en Inglaterra... casi no necesita un objeto.. Su doctrina no es tanto de realidades cuanto de aspectos estereotipados de realidades; por asi decirlo tiene miedo de andar entre lo real... No digo que el asentimiento que inculca y origina no sea genuino con respecto al campo limitado de su doctrina; pero a lo más es nocionals ${ }^{30}$. La que el cardenal inglés advertia en su época ha sido denunciado también en mestro tiempo por el gran biblista alemain $\mathrm{H}$. Schlier, cuando seriala que se está produciendo: «un creciente alejamiento, una extrañeza entre la mentalidad común y la fe cristiana... Para la sensibilidad general y pública, términos cristianos fundamentales y su significado se han vuelto en gran medida incomprensibles». Estando así las cosas, quien quiera usar las palabras cristianas tiene que asumir una tarea hasta ahora inédita: «debe suscitar también el significado de la realidad de la que quiere hablan" ${ }^{37}$. La prueba de que estas preocupaciones no se circunscriben a un grupo minoritario de autores geniales, sino que identifican el problema nuclear de la Iglesia hoy, nos la ofrecen los múltiples textos del Magisterio sobre la useparación fe-vidas ".

5.4 Asi pues, al final de este recortido, aparece un cristianismo dividido, exterior a la razón y a la libertad humanas, que corre el peligro de resultar superfluo para un hombre adulto. Es tan improbable esperar de un cristianismo asi que eduque bien como lo es de la mentalidad moderna en la que se inspira. Si el resultado del proceso educativo moderno-posmoderno es la fragmentación, otro tanto habrá que decir de los procesos de educación en la fe que son culturalmente deudores de esa mentalidad. La proliferación de cursos y cursillos sobre aspectos cada vez más minuciosos de la actividad formativa (análisis de la sociedad, encuestas, presentaciones técnicas de acciones especializadas...), en los que se suele introducir a los educadores, hace pensas que el modelo pedagógico dominante está todavia influido por esta rendencia a concebir la educación como una acumulación de saberes particulares para la formación de expertos.

\footnotetext{
"R. GUARDINI, Freabeit, Jethetesat, Gmade. Munchen, 1948, pp. 9-10.

").H. NEWMAN, El cosentimiento religiose. Barcelona, 1960, pp.78-80.

${ }^{*}$ H. SCHLIER, Linee fondamentali di sma teolagiu pawitina. Brescia, 1995, pp. 12-13.

"DesdeGSN."43aVSN." 88
} 


\section{Javier Prades López.}

\section{Una propuesta que se verifica en la libertad y no una doctrina que se imparte}

Del recorrido hecho hasta ahora se desprende que, frente a la concepción «absoluta» de la razón y la libertad, úpica de la modernidad, el hombre es un sujeto unitario, abierto constitutivamente a la relación de conocimiento y arnor de la realidad, en la que se incrementa toda la personalidad. Es pues necesario advertir que un conocimiento verdadero y objetivo requiere absolutarnente que el hombre entero esté subjetivamente dispuesto a acoger la realidad. Y esto es tanto más necesatio cuando se trata de verdades no puramente especulativas sino relacionadas con el sentido de la vida. Es imposible conocerlas, como diría Tomás, sin una cierta "connaturalidad» con el objeto en cuestión. Por eso, el método que permite esa connaturalidad es el de una verificación, en el tiempo y en el espacio, de una propuesta educativa en la que se es acompañado por quien tiene autoridad moral para keducarn, para introducir en la totalidad de lo real.

6.1 La validez de esa propuesta educativa no se puede determinar a priori. Cada uno de nosotros está llamado a descubrir la verdad de su área del saber y, más radicalmente, la verdad de su propia vida mediante la adhesión a la realidad que le sale al encuentro. Ninguno podemos produciela por entero a partir de nosotros mismos. La verificación de la bondad de este planteamiento no puede consistir en una comparación abstracta entre dos (o varios) sistemas de ideas ya confeccionados, para ver cuál incluye más elementos que los demás y así demostrar su verdad, en un ejercicio de la razón desligado de todo vínculo con la realidad (ab-solutum). Se trata, más bien, de introducirse en la realidad mediante la invitación a comprobar libremente una hipótesis, en el recorrido de un camino humano.

6.2 Los resultados de un proceso educativa no se pueden medir exclusivamente en el plano conceptual, aunque sea imprescindible, sino en la totalidad de la acción humana que es a la vez racional y libre. La libertad no se puede predeterminar sino que se debe ejercitar en cada situación, que se convierte en ocasión de entrat en el misterio de la realidad. Sólo en esta permanente autodererminación libre dél sujeto, gracias a la adhesión a una propuesta, se puede verificar la bondad de un sistema educativo. 


\section{EDUCACIÓN PARA LA LIBERTAD}

\section{El testimonio}

La categoria integral que expresa una posición educativa humana y cristana es la categoria de testimonio ${ }^{\text {th }}$. En este momento debernos limitarnos a esbozar un inicio de respuesta a la pregunta por la dinámica educativa completa. Teniendo en cuenta el contexto en el que nos encontramos, podrá resultar a todos comprensible que adopte el punto de vista de la tradición cristiana y examine el método con el que el Padre celestial ha querido educar a los hombres: Enunt unmes docibiles Dei (cfr.) n. 6, 45).

7.1 Díos no ha querido comunicarse a los hombres a través de una idea sino que ha elegido darse a conocer a rravés de un figura histórica concreta: su Hijo unigénito encarnado por nosotros y por nuestra salvacion.

7.2 En Jesuctisto, la verdad hecha carne ha decidido, a la hora de darse a conocer, pasar a través del acto de libertad de cada hombre. Esto no sígnifica, como es lógico, que la libertad humaria constituya en última instancia la verdad, exponiéndonos al peligro de un relativismo epistemológico. Significa, más bien, que en cada acto de libertad, que es a la vez un acto de comprensión (teórico) y de acción (práctico), la verdad, que es simultáneamente el bien, interpela al yo y le urge a que la reconozca, es decir, le urge a que dé testimonio de ella. Sólo el testimonio, en su consideración más completa, es decir, como característica intrinseca del acto de libertad, puede expresar adecuadamente el encuentro del yo y la realidad. En una época como la nuestra ciertamente hay que decir que sólo el testimonio es digno de crédito. $Y$, por lo que respecta a la vida universitaria, se comprende por qué no se puede realizar si no es de modo comunitario: el encuentro del yo con la realidad exige el testimonio que es el origen de la comunidad. Por eso se puede comprender también la reflexión inicial de Maritain según la cual no se puede reducir la educación a una pura enseñanza. La verdad no se puede comunicar a traves del saber material de un curso. No se pucde prescindir de maestros, de profesores que sean testigos.

\footnotetext{
" Cff. para este epigrafte $A$, SCOLA, aUna experiencia en acto: la Pontificia Universidad Lateranensen, Asociación para la Investigación y la Docencia Universitas, Pryycio sebre aA Andisis...j; at.
} 
7.3 Para evitar cualquier reducción moralista del testimonio a un mero ibuen cjemplon y conservar todo su slcance como categoría educativa, puede ser útil recordat que el testigo es el tercero que se encuentra entre dos y que, como to demuestri el uso juridico de este término, llama a la libertad de ambos al vínculo con la verdad ${ }^{12}$. Así aparece con claridad la tarea que brota de una auténtica relación entre maestro y discipula. El maestro está llamado a ser ese tercero testigo, que mediante su propia experiencia provoca la libertad del estudiante para que se ponga en juego personalmente y dé testimonio de la verdad. También entre profesores, esta dinámica del testimonio es el camino a seguir. El profesor es testigo, permanente, para que en cada acto concreto de libertad del estudiante o de otro colega, estos puedan acceder a la verdad. La verdad no como algo que se puede poseer por completo al modo de un objeto, sino una verdad que se uposee en la distancian.

\section{Una relación entre maestro y estudiantes}

Puede ser útil traer a colación un ejemplo de pasión integral por la realidad, y de profunda conciencia del valor de la relación entre el profesor universitario y sus estudiantes. El Premio Príncipe de Asturias de Humanidades, G. Steiner ha escrito sobre la relación entre docencia e investigación ${ }^{43} y$ ha dejado algunas jugosas anotaciones sobre su experiencia universitaria en Chicago, como estudiante y como joven profesor, que ahora transcribo:

La enseñanza y la camaraderia, la provocación mutua en un seminario, han sido mi oxigeno personal. No puedo imaginar mi obra sin ellas. Si me resisto a jubilarme es porque mis alumnos han sido indispensables. Esto es para mi un tesoro. [...]

El gran maestro está enredado, incluso corponalmente, en el proceso comunicativo y ejemplificador. [...] Una universidad digna es

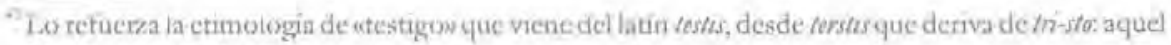
que se pone como tercero.

" d as universidades $\{$, | estín sometidas en fudo momento a tensiones fundumentalex. Ninguna instituciōn angura irganización de enseñanza superior, ba lognado vonciliar de manera
} 


\section{EDUCACIŌN PARA LA LIBERTAD}

sencillamente aquella que propicia el contacto personal del estudiante con el aura y la amenaza de lo sobresaliente. Estrictamente hablando, esto es cuestión de proximidad, de ver y de escuchar... El académico, el profesor, deberian ser perfectamente visibles. Cruzarse a diario en nuestro camino... En virtad de esta contigüidad no forzada, el estudiante, el joven investigador quedará (o debería quedar) infectado. Percibirá el perfume de lo real. Recurro al uso de términos sensoriales porque el impacto puede ser fisico, [...] Una vez que un hambre o uns mujer jóvenes son expuestos al virus de la absoluto, una vez que ven, oyen, "huelen" la fiebre en quienes persiguen la verdad desinteresada, algo de su resplandor permanecerá en ellos. [...]

Corri a la biblioteca. Esa noche intenté hincarle el diente al primer parrafo de Sery tiempo. Era incapaz de entender incluso la frase más breve y aparentemente directa. Pero el torbellino ya había comenzado a girar; el presentimiento radical de un mundo absolutamente nuevo para mí. Prometi intentarlo una vez más. Y otra. Ésa es la cuestión. Llamar la atención de un estudiante hacia aquello que, en un principio, sobrepasa su entendimiento, pero cuya estatura y fascinación le obligan a persistir en elintento $[\ldots . .10)^{-4}+$

Cuando un hombre ba quedado fascinado por to que sobrepasa su entendimiento, durante su época de estudiante, y cultiva esa apertura de la razón a la misteriosa profundidad de lo real, puede convertirse, en el contacto personal con sus estudiantes, en un "propagadon" de esta beneficiosa fiebre de curiosidad y deseo que alientan toda verdadera actividad universitaria. El profesor Steiner enseñaba en la muy bien pertrechada Universidad de Chicago y, no obstante, no

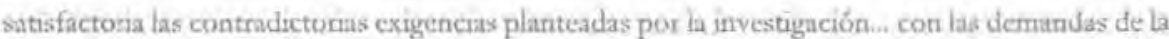
educaciún general y la formación civica. $\{\ldots$, Los nbjetives del sabio humanista, del pensador especulativo \{... $\}$ coineaden de manera imperfecta, si es que cuiaciden de algin mudo, con la tarea del pedagogo, del instructor general. I... I En términos ideales, la trutición fuef pande quedar fuera de la actividad docente. Estoy convencido de que deberiay. G. STEINTK, Emifa, Madrid, 1998, pp. $57-67$.

"STEINER, Emsta. L, $c$ 


\section{Javier Prades López}

duda en privilegiar estas dimensiones personales de la docencia antes que la sobreabundancia de medios materiales.

\section{Conclusión}

Se comprende entonces por qué la educación completa requiere que todos los recursos materiales y pedagógicos en si irrenunciables faciliten la relación personal entre los docentes y de estos con los estudiantes. Es una relación que se cualifica existencialmente como testimonio, en cuanto que hace posible que el interlocutor esté ante el encuentro con la verdad. De este modo se puede verificar la verdad de esa propuesta educativa en el ejercicio de la libertad, y alcanzar así el éxito tan deseado como infrecuente de la vida académica. Lat experiencia multidisciplinar e interuniversitaria de la Asociación para la Investigación y la Docencia "Universitas” en España es un intento de ayuda entre profesores y doctorandos para verificar la conveniencia de esta propuesta ${ }^{45}$.

\footnotetext{
"Màs información sobre ia Asociación Dniverstas en wwwasociacion-universutase.
} 